\title{
Tratamiento nutricional con dieta cetogénica en niños con epilepsia refractaria - revisión narrativa
}

\author{
Nutritional treatment with ketogenic diet in children with \\ refractory epilepsy - review \\ Tratamento nutricional com dieta cetogênica de crianças com \\ epilepsia refratária - revisão narrativa
}

Sara Valentina Acuña Escobar', Carolina Ardila', Sayda Milena Pico Fonseca ${ }^{*}$

Recibido: 9 de octubre de 2019. Aceptado para publicación: 15 de mayo de 2020

Publicado en línea: 2 de junio de 2020

https://doi.org/10.35454/rncm.v3n2.108

\section{Resumen}

Introducción: la epilepsia refractaria es definida como aquella en la que las crisis recurrentes no permiten a quienes la padecen vivir plenamente, o bien porque el tratamiento anticonvulsivante que recibe el paciente no logra controlar las crisis o debido a los efectos secundarios de los medicamentos. La dieta cetogénica aparece en 1920 gracias al efecto antiepiléptico del ayuno. Esta dieta reproduce los efectos metabólicos del ayuno, obligando al cuerpo humano a utilizar la grasa como fuente de energía. El presente artículo tiene como objetivo revisar la evidencia científica de la acción específica de la dieta cetogénica en la epilepsia refractaria.

Método: se realizó una revisión narrativa, con búsqueda no sistemática de artículos relevantes en español e inglés utilizando las palabras clave epilepsia, epilepsia en niños, epilepsia refractaria en edad pediátrica, dieta cetogénica. Las bases de datos consultadas fueron PubMed, Scielo, BVS, Redalyc, Latindex y Google académico, se incluyeron artículos publicados en los últimos 20 años.

Resultados: el efecto de la dieta cetogénica está relacionada con el mecanismo de utilización de los ácidos grasos. Se encontraron cuatro diferentes tipos de dietas cetogénicas usadas, las cuales manejan distintos rangos de relación gramos de

\section{Summary}

Introduction: Drug-resistant epilepsy is defined by the occurrence of persistent seizures that do not allow those suffering them to lead a fulfilling life, either due to antiepileptic drug treatment failure or their side effects. The ketogenic diet appeared in 1920 after observing that fasting had an antiepileptic effect. This diet induces the same metabolic effects as fasting, forcing the human body to use fat as a source of energy. The objective of this article is to assess the available scientific evidence on the specific action of a ketogenic diet on refractory epilepsy.

Methods: We performed a non-systematic search in PubMed, Scielo, BVS, Redalyc, Latindex, and Google Scholar for articles in Spanish and English published in the last 20 years using the keywords epilepsy, epilepsy in children, refractory epilepsy in pediatric age, and ketogenic diet.

Results: The antiepileptic effect seems to be related to fatty acid metabolism. Four different types of ketogenic diets with differing fat-to-protein ratios, but all with the purpose of keeping the patient in a state of ketosis. The metabolic and neurophysiological effects caused by the ketogenic diet in children with refractory epilepsy have only been described in animal experimental studies, not in human studies.

\section{Resumo}

Introdução: epilepsia refratária é definida como aquela em que as crises recorrentes não permitem que o paciente viva plenamente ou porque o tratamento anticonvulsivante falha em controlar as crises ou devido aos efeitos secundarios dos medicamentos. A dieta cetogênica surgiu em 1920, graças ao efeito antiepilético do jejum. Esta dieta reproduz os efeitos metabólicos do jejum, forçando o corpo humano a usar a gordura como fonte de energia. Este artigo tem como objetivo rever a evidência científica da ação específica da dieta cetogênica na epilepsia refratária.

Método: foi realizada uma revisão narrativa, com busca sistemática de artigos relevantes em espanhol e inglês, utilizando as palavras-chave epilepsia, epilepsia em crianças, epilepsia refratária em idade pediátrica, dieta cetogênica. As bases de dados consultadas foram: PubMed, Scielo, BVS, Redalyc, Latindex e Google acadêmico, foram incluídos os artigos publicados nos últimos 20 anos.

Resultados: o efeito da dieta cetogênica está relacionado com o mecanismo de uso dos ácidos graxos. Se encontraram quatro tipos diferentes de dietas cetogênicas, que lidam com diferentes proporções de grama de gordura por grama de proteína; embora todos com o mesmo objetivo 
grasa por gramos de proteína; aunque todas con la misma finalidad de mantener al paciente en un estado de cetosis. Los efectos, tanto metabólicos como neurofisiológicos, provocados por la dieta cetogénica en niños con epilepsia refractaria solo se han descrito en estudios experimentales en animales, no en humanos.

Conclusión: la evidencia científica actual muestra resultados prometedores en el uso de la dieta cetogénica en la epilepsia refractaria. Sin embargo, el número limitado de estudios y del tamaño de la muestra dio como resultado una deficiente calidad general de la evidencia.

Palabras clave: epilepsia refractaria, dieta cetogénica, niños.
Conclusion: The current scientific evidence shows promising results for the use of a ketogenic diet in children with refractory epilepsy. However, the limited number of studies and sample size yield yield a low overall quality of evidence.

Keywords: Drug Resistant Epilepsy; Diet ketogenic; Children. de manter o paciente em um estado de cetose. Os efeitos metabólicos e neurofisiológicos causados pela dieta cetogênica em crianças com epilepsia refratária foram descritos apenas em estudos experimentais em animais e não em humanos.

Conclusão: as evidências científicas atuais mostram resultados promissores no uso da dieta cetogênica na epilepsia refratária. No entanto, o número limitado de estudos e o tamanho das amostras resultaram em uma deficiente qualidade geral da evidência.

Palavras-chave: epilepsia refratária, dieta cetogênica, crianças.
'Institución Universitaria Escuela Nacional del Deporte, Cali, Colombia

\section{INTRODUCCIÓN}

La epilepsia es el trastorno neurológico crónico más común. Se presenta en $0,5 \%-1 \%$ de la población mundial y en $60 \%$ de los casos se inicia en la infancia ${ }^{(1)}$. Según un estudio epidemiológico que se llevó a cabo en las ciudades de Bogotá, Medellín, Bucaramanga y Cartagena se reportó una prevalencia de $10,3 \%{ }^{(2)}$. Su origen está asociado a descargas eléctricas excesivas de las neuronas, produciendo crisis asumidas de diferentes formas que van desde pérdidas repentinas de la conciencia hasta convulsiones graves y frecuentes ${ }^{(3-5)}$. El tratamiento de esta enfermedad es farmacológico. La epilepsia refractaria o también conocida como farmacorresistente está definida como aquella en la que las crisis son tan recurrentes que impiden que el paciente pueda vivir plenamente, o también cuando el tratamiento anticonvulsivante no logra controlar las crisis o por sus efectos secundarios ${ }^{(2)}$. Aproximadamente $30 \%$ de los pacientes con epilepsia de cualquier tipo persistirá con crisis epilépticas a pesar del manejo farmacológico $^{(6)}$. En estos pacientes el tratamiento dietético se ha planteado como una alternativa terapéutica o como una herramienta coadyuvante ${ }^{(7)}$.

La dieta cetogénica (DC) y el efecto epiléptico del ayuno aparece inicialmente en 1920. Esta dieta repro-
*Correspondencia: Sayda Milena Pico Fonseca sayda.pico@endeporte.edu.co duce los efectos metabólicos del ayuno, obligando al cuerpo humano a utilizar la grasa como fuente de energía. El mecanismo de acción por el cual la DC funciona como antiepiléptico aún permanece en investigación. La principal teoría se basa en que los cuerpos cetónicos (acetoacetato, acetona y beta-hidroxibutirato), sintetizados en el hígado a partir de ácidos grasos de cadena larga y media, son anticonvulsivos directos. $\mathrm{Al}$ atravesar la barrera hematoencefálica funcionan como fuente primaria de energía e inducen un estado de cetosis, momento en el cual hay mayor eliminación de glutamato, disminución de su transporte vesicular y aumento de la conversión de este neurotransmisor a $\mathrm{GABA}^{(8)}$. Con la aparición de nuevos anticonvulsivantes en 1938, la DC es relegada hasta hace 20 años cuando resurge y adquiere importancia en el tratamiento de la epilepsia refractaria a los fármacos ${ }^{(2)}$.

La evidencia muestra que la DC, y sus variantes, son una buena alternativa para pacientes de cualquier edad, farmacorresistentes no quirúrgicos con epilepsia, teniendo en cuenta que el tipo de dieta debe diseñarse individualmente y que las dietas menos restrictivas y más apetecibles suelen ser mejor opción para adultos y adolescentes. Por lo tanto, el objetivo de la presente revisión es mostrar la evidencia actual sobre los posibles mecanismos de acción, su aplicabilidad y eficacia ${ }^{(9,10)}$. 


\section{MÉTODO}

\section{Estrategias de búsqueda}

Se hizo una búsqueda en las bases de datos SciELO, Medline, PubMed, Google académico de estudios publicados desde el año 1998 hasta 2019, en español, portugués e inglés utilizando epilepsia en niños, epilepsia refractaria en edad pediátrica, dieta cetogénica como palabras claves.

Se incluyeron los estudios de pacientes en edad pediátrica con diagnóstico médico de epilepsia refractaria o resistente a los fármacos. Además, estudios que mencionen tratamientos para la epilepsia refractaria en niños, en los que se describen los métodos y herramientas utilizadas.

\section{Tratamiento nutricional con dieta cetogénica en niños con epilepsia refractaria}

En el tratamiento de la epilepsia refractaria se contemplan el manejo farmacológico y quirúrgico como las principales alternativas; sin embargo, la inclusión de un régimen dietario especial denominado dieta cetogénica, presenta nueva evidencia de posibles efectos positivos en las áreas cognitiva y conductuales en pacientes pediátricos con epilepsia. Algunos autores han referido mejor estado de ánimo, menor nivel de ansiedad, mayor grado de atención y concentración, mayor vocabulario receptivo y mejor velocidad en el procesamiento de la información en niños con episodios epilépticos manejados con dieta cetogénica ${ }^{(11,12)}$.

\section{Mecanismo de acción}

La dieta cetogénica hace referencia a una ingesta alta de grasas, sustituyendo en gran medida los carbohidratos y conservando las proteínas. Esta modificación dietaria genera cambios metabólicos principalmente la cetosis, la disminución de glucosa, el aumento de niveles de ácidos grasos libres, situación similar a la observada en los procesos de inanición ${ }^{(13-15)}$.

El control de las convulsiones parece estar dado directa o indirectamente por los cuerpos cetónicos, los ácidos grasos libres (principalmente poliinsaturados) y la restricción de la glucosa. Aunque no se tiene certeza sobre el mecanismo exacto se piensa que estos tres elementos son necesarios para que esta dieta sea efectiva. Además, un aumento en la biogénesis mitocondrial, la fosforilación oxidativa, los mejores niveles de ácido gamma-amino butírico (GABA), la reducción de la excitabilidad y la activación neuronales y la estabilización de la función sináptica son posibles mecanismos de acción ${ }^{(16)}$.

Por lo tanto, los posibles efectos terapéuticos se han atribuido a cambios en el funcionamiento celular, en los que, la acción de los componentes grasos y su influencia llevan a una menor excitabilidad neuronal. Es posible que estos efectos sean generados por un cambio metabólico que tiene lugar en la célula, en el que se deja de utilizar glucosa como fuente primaria de energía para obtener adeniltrifosfato a partir de cuerpos cetónicos. Esta idea se postula basada en la gran variedad de epilepsias en las que se ha indicado dieta cetogénica y se han observado efectos benéficos bastante favorables, reforzando así la hipótesis de su acción en múltiples niveles y no en un punto único del metabolismo neuronal ${ }^{(17,18)}$.

En relación con los efectos a largo plazo de la DC sobre la célula, se identifican varios mecanismos que pudieran tener un rol activo. La cetosis reduce la producción de radicales libres (ROS), y el estrés oxidativo mejora el estado redox, promueve la biogénesis mitocondrial, e incrementaría la expresión de proteínas específicas del metabolismo energético. Los cuerpos cetónicos al parecer disminuyen la concentración de ROS, pues mejorarían la síntesis de glutatión mitocondrial, actuando directamente sobre factores de transcripción ${ }^{(2,12,17)}$.

Los cuerpos cetónicos acetoacetato y acetona han demostrado tener un papel en la prevención de las crisis gatilladas por pentilentetrazol en ratas, y se ha observado una modulación de la actividad gabaérgica en neuronas de la sustancia negra (mediante canales de potasio sensibles a ATP), con el consiguiente efecto sobre áreas epileptógenas corticales ${ }^{(19)}$.

Durante la infancia el cerebro es más eficiente en la extracción y utilización de cuerpos cetónicos de la sangre, debido a los niveles más altos de enzimas que metabolizan las cetonas y los transportadores de ácido mono carboxílico producidos en esta dieta. La cantidad de dichos transportadores disminuye a medida que el cerebro madura, pero siguen estando presentes en la edad adulta ${ }^{(20)}$.

Aunque en la literatura se encuentran distintos tipos de DC todas comparten el objetivo de controlar la epilepsia y al mismo tiempo aumentar la adherencia, mejorando la palatabilidad, la diversidad de alimentos y minimizando la aparición de efectos adversos. En la DC clásica (4:1), las grasas proporcionan aproximadamente $90 \%$ de la energía ingerida (en su mayoría triglicéridos de cadena larga), las proteínas $8 \%$ y los carbohidratos $2 \%$. La dieta debe estar ajustada a los requerimientos de cada paciente ${ }^{(21)}$ (Tabla 1$)$. 
Tabla 1. Composición de los diferentes tipos de dieta cetogénica y la relación cetogénica

\begin{tabular}{|l|c|c|c|c|c|}
\hline \multicolumn{1}{|c|}{ Tipo de dieta } & $\begin{array}{c}\text { Grasa de } \\
\text { alimentos \% VCT }\end{array}$ & TCM \% VCT & Proteínas \% VCT & Carbohidratos \% VCT & $\begin{array}{c}\text { Relación } \\
\text { cetogénica }\end{array}$ \\
\hline Clásica 4:1 & 90 & - & 10 & 10 & $4: 1$ \\
\hline Clásica 3:1 & 87 & - & 13 & 13 & $3: 1$ \\
\hline Combinada TCM & 11 & 60 & 10 & 19 & $1,2: 1$ \\
\hline Atkins modificada & 41 & 30 & 10 & Alimento bajo índice & $1,2: 1$ \\
\hline Bajo índice glucémico & $35-40$ & - & $15-20$ & 19 \\
\hline
\end{tabular}

VCT: valor calórico total. TCM: triglicéridos de cadena media.

En este tipo de dieta, también pueden ser utilizados los triglicéridos de cadena media (TCM), por lo que recibe el nombre de DC con TCM. Los TCM son absorbidos y oxidados más rápidamente en comparación con los triglicéridos de cadena larga (TCL) debido a su facilidad de atravesar en forma pasiva la membrana celular. Estas grasas también tienen una capacidad única de promover la síntesis de cuerpos cetónicos en el hígado. Por lo tanto, agregar TCM a la DC permite aumentar la cantidad de carbohidratos lo que facilita una mayor adherencia al tratamiento dietario ${ }^{(22)}$.

Es imprescindible la presencia de ácidos grasos esenciales en la dieta, aunque el uso de estos puede traer consecuencias de tipo gastrointestinal como diarrea y sensación constante de saciedad, que podrían restringir su uso. De esta forma se propone el uso de este tipo de dieta junto con el de la DC clásica consiguiendo así mayor aceptación y eficacia con menos restricciones que las dietas antes mencionadas ${ }^{(23)}$.

Otro tipo de dieta cetogénica es la dieta Atkins modificada que permite el consumo de proteínas y lípidos, restringiendo carbohidratos y aumentándolos progresivamente. Además, a la dieta Atkins es posible agregarle un preparado similar al de la DC clásica, y así contribuir al aumento de la relación cetogénica y por ende la cetosis.

La dieta Atkins modificada restringe los carbohidratos a 10 gramos / día en niños (15 gramos / día en adultos), en esta dieta se ajustan las grasas de 1 a 1 (gramos de grasa combinada de proteínas y carbohidratos), con aproximadamente $65 \%$ de las calorías provenientes de fuentes de grasas; se inicia sin ayuno o admisión, y los carbohidratos se limitan a 10 a 20 gramos por día. Se han realizado estudios usando esta dieta en más de 450 niños, adolescentes y adultos, los cuales revelan que $45 \%$ tiene una reducción de las convulsiones de $50 \%$ - $90 \%$, y un poco más de $25 \%$ tiene una reducción de las convulsiones superior a $90 \%{ }^{(24)}$.

Por otro lado, la dieta de bajo índice glicémico cuyo objetivo es conservar estables los niveles de glucosa en sangre, también hace parte de las dietas cetogénicas en la epilepsia refractaria. Esta dieta se caracteriza por permitir un mayor aporte de carbohidratos en comparación con la DC clásica, DC con TCM y con la Atkins modificada. Es necesario controlar estrictamente la cantidad de carbohidratos brindados, y escoger alimentos que posean un índice glucémico inferior a 50, prefiriendo alimentos con alto porcentaje de fibra ${ }^{(25,26)}$.

En la Tabla 1 se presentan diferentes modelos de la composición de macronutrientes de la dieta cetogénica, y su valor calórico total.

Para seleccionar el tipo de dieta, es necesario tener en cuenta la edad del niño, el tipo y gravedad de la epilepsia, la necesidad de obtener una respuesta oportuna, características de la familia, los hábitos alimentarios del niño y la disponibilidad de personal entrenado (Nutricionista) para la elaboración de las dietas ${ }^{(22)}$.

\section{Dieta cetogénica clásica vs Dieta Atkins modificada}

Indicada para niños menores de 2 años. En un ensayo se comparó la eficacia, seguridad y tolerancia de la DC clásica con la Atkins modificada en niños con epilepsia refractaria. La Atkins modificada se consideró la opción principal para el tratamiento de la epilepsia refractaria en niños, pero la DC clásica es más adecuada en primera línea de terapia de dieta en pacientes $<2$ años $^{(26)}$. Otro estudio reportó una mejoría muy importante en aquellos pacientes que padecían epilepsia mioclónica, 
valoró la respuesta frente al tratamiento con dieta Atkins y posterior cambio a DC clásica encontrando una mejoría muy importante, por lo que se sugiere iniciar el tratamiento con la DC clásica ${ }^{(27)}$.

La evidencia muestra que los niños pueden mantener el control de las convulsiones cuando la DC clásica (4:1) se cambia por la dieta de Atkins modificada ${ }^{(26)}$. Un estudio evaluó la probabilidad de un control adicional de las convulsiones con un cambio de Dieta Atkins modificada a DC clásica (4:1). Los resultados sugieren que la DC 4:1 probablemente tenga más efecto que la Atkins modificada lo que puede beneficiar a las personas con epilepsia mioclónica-astática en particular. Se evidenció una respuesta mucho más favorable al usar la DC clásica en pacientes con soporte nutricional enteral ${ }^{(26)}$.

\section{Dieta Atkins modificada}

Indicada en pacientes entre los 2 y los 6 años. La evidencia muestra que cuando se compara la DC clásica con la dieta Atkins modificada, la respuesta es la esperada; en adolescentes y adultos, se sugiere aumentar proporcionalmente la cantidad de grasa brindada por medio de un preparado líquido similar a la DC clásica al iniciar el tratamiento dietario y durante el primer mes ${ }^{(25)}$.

\section{Dieta de bajo índice glucémico}

Esta dieta no requiere realmente una estricta planeación y diseño, pero se recomienda su uso en las siguientes situaciones: cuando no se cuente con la disponibilidad del profesional especializado para diseñar una DC clásica; al no haber tolerancia o adherencia a las dietas más restrictivas; y mientras el paciente espera iniciar la DC clásica ${ }^{(24)}$.

\section{DISCUSIÓN}

\section{Evidencia de la eficacia de la dieta cetogénica}

Vaccarezza y colaboradores analizaron en forma retrospectiva cinco historias clínicas de niños entre 1 y 14 años, tres de ellos con diagnóstico de FIRES (febrile infection-related epilepsy syndrome) y dos con diagnóstico de epilepsia parcial sintomática refractaria. Todos los pacientes recibieron diferentes tratamientos terapéuticos, pero ante el fracaso de estos, decidieron utilizar la dieta cetogénica tradicional ${ }^{(13)}$. Cuatro pacientes, después de iniciar la dieta cetogénica, manifestaron la disminución de los síntomas presentando buena tolerancia. Uno de los pacientes no presentó respuesta y murió. En pacientes que presentan epilepsia refractaria, ante el fallo de los fármacos anti convulsionantes, el uso de la dieta cetogénica podría ser aceptado, dada su alta efectividad y seguridad ${ }^{(13)}$.

Un estudio prospectivo que midió el impacto nutricional de la dieta, incluyó 12 pacientes con edades entre 4 y 17 años y la utilización de dieta cetogénica durante un mínimo de 6 meses y un máximo de 12 meses $^{(11)}$; los pacientes fueron ingresados en un hospital para el ayuno de inducción a la cetosis, recibieron suplemento de vitaminas y minerales, y seguidos de manera ambulatoria. Los resultados no mostraron cambios hematimétricos, a excepción de un paciente que presentó hipercloremia sin consecuencias clínicas, las convulsiones disminuyeron en promedio $56 \%$ a los 6 meses de uso de la dieta, y $80 \%$ en quienes la utilizaron durante 12 meses. Con la dieta cetogénica se consiguió un mayor control en pacientes con epilepsia refractaria a los fármacos, sin alterar el crecimiento o causar efectos secundarios importantes ${ }^{(11)}$.

Una revisión retrospectiva de historias clínicas de 41 niños que tenían epilepsia refractaria tratados con dieta cetogénica, la mayoría con dieta tipo Radcliffe II, con una edad media de inicio de la dieta de 3,9 años, y seis meses después de iniciar la dieta, mostró que 10,5\% alcanzó más de $90 \%$ de reducción de las crisis y 5,6\% no volvió a presentar crisis. En menos del $4 \%$ de los individuos se evidencio la existencia de efectos secundarios tolerables y transitorios como aumento de los niveles de colesterol y estreñimiento, sin variación de los parámetros antropométricos. La dieta cetogénica supone una alternativa terapéutica oportuna en el manejo de la epilepsia refractaria en la edad pediátrica, existiendo una mayor probabilidad de beneficios cuando se inicia la terapia nutricional a menor edad. Pese a que en general es un tratamiento bien tolerado, se sugiere control nutricional periódico ${ }^{(12)}$.

En otro estudio evaluaron la eficacia y tolerancia de la dieta cetogénica en el manejo terapéutico de niños con epilepsia refractaria, para los criterios de inclusión se tuvieron en cuenta los pacientes con epilepsia resistente al tratamiento, con uno o más fármacos antiepilépticos (FAE); con rangos séricos de FAE en niveles terapéuticos; ausencia de enfermedad hepática, renal, anormalidades metabólicas o errores innatos del metabolismo $\mathrm{u}$ otras encefalopatías progresivas; del mismo modo, las familias debían estar en condiciones psicológicas y económicas para cumplir estrictamente la dieta cetogénica. Todos los pacientes fueron sometidos a examen físico, neurológico, análisis sanguíneos, incluido perfil lipídico 
antes y durante la dieta cetogénica. Se evaluó la eficacia de la dieta cetogénica con base en el porcentaje de reducción de la frecuencia de las crisis (fueron positivos cuando la reducción fue de $50 \%$ o más). Se tomó en consideración la aceptación de la dieta y la calidad de vida. Se admitieron 18 pacientes con un rango de edad entre 2 y 11 años, diez de ellos de sexo masculino y ocho de sexo femenino. Los diagnósticos de los distintos tipos y síndromes epilépticos se realizaron de acuerdo con la clasificación de la ILAE (1989). Finalmente, la dieta cetogénica fue bien tolerada en 14 de los 18 pacientes y sus familias. Siete de los 14 pacientes en tratamiento de dieta cetogénica mostraron una mejoría en la frecuencia de aparición de las crisis y en la calidad de vida. Debido al bajo número de pacientes, $y$ al corto tiempo de seguimiento, no se logró relacionar los resultados con cada uno de los síndromes epilépticos, ni con el riesgo de complicaciones tardías ${ }^{(28)}$.

La Tabla 2 muestra las recomendaciones generales de la terapia con dieta cetogénica.

Tabla 2. Recomendaciones

El tratamiento nutricional con dieta cetógenica debe cumplirse con rigurosidad y responsabilidad para que el efecto anticonvulsivo sea mantenido.

Luego de unos meses de uso de la dieta cetogénica y de observar la efectividad de la dieta, se puede considerar la disminución de la relación cetogénica con el fin de conseguir una mejor adherencia.

Cuando el uso de la dieta cetógenica presenta resultados eficaces, esta puede ser usada hasta por dos años.

\section{Resumen de resultados}

Actualmente la dieta cetogénica como tratamiento para la epilepsia refractaria ha demostrado resultados positivos en estos pacientes; sin embargo, sus efectos continúan siendo motivo de estudio. Existen diferentes ratios de dieta cetogénica, y dado que cada paciente es diferente se deben tener en cuenta estos ratios de manera individual; la evidencia muestra que la disminución de este ratio podría aumentar la adherencia a la dieta cetogénica.

Los principales efectos adversos relacionados con la dieta cetogénica son de poca gravedad y fundamentalmente se trata de alteraciones metabólicas como la hiperuricemia, hipercolesterolemia, hipocalcemia, hipomagnesemia y acidosis. Los triglicéridos de cadena media generan mayor cantidad de cetonas por caloría que los triglicéridos de cadena larga, permitiendo el consumo de una mayor cantidad de proteínas y carbohidratos ${ }^{(28)}$.

\section{CONCLUSIONES}

Los efectos tanto metabólicos como neurofisiológicos provocados por la dieta cetogénica en epilepsia refractaria han sido descritos en estudios experimentales en animales, no en humanos. Sin embargo, varios estudios han demostrado que aproximadamente la mitad de los pacientes disminuyen $50 \%$ el número de convulsiones, y cerca de un tercio del total disminuye en $90 \%$ el número de crisis.

La presente revisión de la evidencia científica muestra resultados prometedores en el uso de la DC en la epilepsia refractaria. Sin embargo, el número limitado de estudios y los tamaños de muestra pequeños dieron como resultado una calidad general de la evidencia deficiente. Existen otras dietas menos rigurosas y más fáciles de consumir, como la dieta Atkins modificada o la de TCM, que pueden tener un efecto similar al de la DC clásica en el control de las convulsiones, pero esta suposición requiere mayor investigación. Para los pacientes pediátricos con epilepsia medicamente intratable o que no son candidatos a intervención quirúrgica, la $\mathrm{DC}$ sigue siendo una opción válida, aunque requiere más investigación.

Para la implementación de la DC se necesita un equipo médico multidisciplinario especializado conformado por especialistas en nutrición y neurología, para un adecuado seguimiento de los pacientes y para lograr una buena adhesión al tratamiento.

\section{Financiación}

El presente trabajo se encuentra financiado por la Institución Universitaria Escuela Nacional del Deporte.

\section{Declaración de conflicto de intereses}

Las autoras declaran la inexistencia de conflictos de interés en relación con este artículo.

\section{Declaración de autoría}

Los autores contribuyeron en partes iguales a la escritura, revisión y documentación de la presente revisión de tema. 


\section{Referencias bibliográficas}

1. Hauser AW, Hesdorffer CD, Epilepsy Foundation of America. Epilepsy: frequency, causes and consequences. First Edition. New York: Demos Medical Pub; 1990.

2. Carrillo A, Jiménez V, Loubat M, Castillo R, Marín F, Carvajal $\mathrm{M}$, et al. Estudio descriptivo-comparativo de la calidad de vida de niños, entre 6 y 10 años, con epilepsia refractaria y no refractaria al tratamiento. Revista Chilena de Epilepsia. 2002; 3(3):4-10.

3. Bustacara Dl, Norela CL, Ladino ML. Manejo nutricional con dieta cetogénica en niños con epilepsia refractaria. Rev Gastrohnup. 2012; 14(3): 123-7.

4. Barañano WK, Hartman LA. The Ketogenic Diet: Uses in Epilepsy and Other Neurologic Illnesses. Curr Treat Options Neurol. 2008;10(6):410-9. doi: 10.1007/s11940-008-0043-8

5. Chang SB, Lowenstein HD. Epilepsy. N Engl J Med. 2003;349:1257-66. doi: 10.1056/NEJMra022308.

6. Fundación Liga Central Contra la Epilepsia. ¿Qué es la epilepsia? [Internet]. Bogotá, Colombia: Fundación Liga Central Contra la Epilepsia [citado 11 de mayo de 2020]. Disponible en: http://www.epilepsia.org/epilepsia/que-es-epilepsia/.

7. Conejo MD. Características clínicas, etiología, tratamiento y evolución de la epilepsia refractaria en la infancia [Tesis Doctoral] [Internet]. Valladolid, España: Universidad de Valladolid; 2015. [citado 11 de mayo de 2020]. Disponible en: http://uvadoc.uva.es/handle/10324/16219.

8. Moog CJ, Ochoa CW. ¿Qué es la epilepsia refractaria? IATREIA: 2003;16(2):163-7.

9. Avanzini G. Molecular and Cellular Targets for Anti-epileptic Drugs. London, England: John Libbey: 1997.

10. Proposal for classification of epilepsies and epileptic syndromes. Commission on Classification and Terminology of the International League Against Epilepsy. Epilepsia. 1989;30(3):389-99. doi: 10.1111/j.1528-1157.1989.tb05316.x.

11. Hernández-Vicente M, García GP, Nagel GA, Muñoz LF, Álamo C. Abordaje terapéutico desde la perspectiva nutricional: situación actual del tratamiento dietético. Neurología. 2007; 22(8): 517-25.

12. Pedrón GC. Epilepsia y alimentación. Dietas cetogénicas. Nutr Hosp Suplementos. 2009; 2(2):79-88.

13. Vaccarezza M, Silva W, Maxit C, Agosta G. Super-refractory status epilepticus: treatment with ketogenic diet in pediatrics. Rev Neurol. 2012;55(1):20-5.

14. Appavu B, Vanatta L, Condie J, Kerrigan JF, Jarrar R. Ketogenic diet treatment for pediatric super-refractory status epilepticus. Seizure. 2016;41:62-5. doi: 10.1016/j.seizure.2016.07.006. Epub 2016 Jul 21.

15. O'Connor SE, Ream MA, Richardson C, Mikati MA, Trescher $\mathrm{WH}$, Byler DL, et al. The ketogenic diet for the treatment of pediatric status epilepticus. Pediatr Neurol. 2014;50(1):1013. doi: 10.1016/j.pediatrneurol.2013.07.020.

16. Cobo NH, Sankar R, Murata KK, Sewak SL, Kezele MA, Matsumoto $\mathrm{JH}$. The ketogenic diet as broad-spectrum treatment for super-refractory pediatric status epilepticus: challenges in implementation in the pediatric and neonatal intensive care units. J Child Neurol. 2015;30(2):259-66. doi: 10.1177/0883073813516192.

17. Ramos FAM. Impacto nutricional da dieta cetogênica na epilepsia refratária infantil de difícil controle [Tesis de posgrado en Neurología] [Internet]. São Paulo, Brasil: Universidad Federal São Paulo; 2004 [citado 11 de mayo de 2020]. Disponible en: http://repositorio.unifesp.br/handle/11600/20391

18. Sánchez-Pablos T, Leal-Oliveros L, Núñez-Enamorado N, Camacho-Salas A, Villares-Moreno JM, De las Heras RS. Experiencia en el tratamiento con dieta cetogénica de la epilepsia refractaria en la edad pediátrica. Rev Neurol. 2014; 58:55-62. DOI: https://doi.org/10.33588/rn.5802.2013440.

19. Calderón BB, Sión BE. Epilepsia y fármacos antiepilépticos: ¿Cuánto hemos avanzado en estos últimos 20 años? Revista Chilena de Epilepsia. 2019; 19(2): 14-8.

20. García-Peñas JJ. Trastornos del espectro autista y epilepsia: el papel de la dieta cetogénica. Rev Neurol. 2016; 62(supl.1): S73-S78. DOI: https://doi.org/10.33588/rn.62S01.2015525

21. Palacios SL. Epilepsy. Rev Colomb Psiquiatr. 1999;28(2):14656.

22. López-Pisón J, García OA, Sáenz de CA, Cáceres A, Eiras J, Bertol V, et al. Epilepsias refractarias en pediatría. Diagnóstico y tratamiento. Acta Pediatr Esp. 2007;65(4):157-64.

23. Berg AT, Shinnar S, Levy SR, Testa FM, Smith-Rapaport S, Beckerman B. Early development of intractable epilepsy in children: a prospective study. Neurology. 2001;56(11):144552. doi: 10.1212/wnl.56.11.1445.

24. Wheless JW, Kossoff EH. Ketogenic Diets. En: Swaiman KF, Ashwal S, Ferriero DM, Schor NF, Finkel RS, Gropman AL, et al. Swaiman's Pediatric Neurology. Principles and Practice. 6th Edition. Elsevier; 2017 p. 624-30.

25. Crumrine PK. Antiepileptic Drug Selection in Pediatric Epilepsy. J Child Neurol. 2002;17(Suppl 2): 2S2-2S8. doi: $10.1177 / 08830738020170020701$.

26. Vivir con epilepsia. Guía de apoyo para niños y adolescentes con epilepsia [Internet]. Febrero 16 de 2016. [citado 12 de mayo de 2020]. Disponible en: https://vivirconepilepsia.es/ guia-de-apoyo-para-ninos-y-adolescentes-con-epilepsia.

27. Pharm GWR. Antiepileptic Drug Treatment: Outcomes and Adherence. Pharmacotherapy. 2000; 20(8): 191S-199S. https://doi.org/10.1592/phco.20.12.191S.35250.

28. Wyllie E. Surgical treatment of epilepsy in children. Pediatr Neurol. 1998;19(3):179-88. doi: 10.1016/s08878994(98)00062-9. 